\title{
MANZANAS PARA CÍDIPE Y ATALANTA: ¿OBSEQUIO AMOROSO O ESTRATEGIA PARA EL ENGAÑO?
}

\author{
Apples for Cydippe and Atalanta: a love gift or a deceit strategy?
}

\author{
Abigail Luz Marina Méndez* \\ Universidad Nacional del Nordeste \\ abigailluzmarinamendez@gmail.com
}

Palabras clave

Ovidio; mito;

manzana; engaño;

intertextualidad

\section{RESUMEN}

Ovidio utiliza la manzana como elemento simbólico para referir las historias amorosas de Cídipe y Aconcio, en las Heroidas 20 y 21, y de Atalanta e Hipómenes, en Metamorfosis 10 (560-707). En ambos casos la unión de las parejas se produce a partir de un engaño o estratagema, cuyo detonante -la manzana- condiciona las formas de representación de las figuras femeninas. En este trabajo abordaremos la resignificación simbólica de la manzana en la presentación de ambos mitos, no solo como componente argumental, sino también como parte estructural de las narraciones ovidianas, en tanto estrategia narrativa ambigua, ya que, si bien su significado responde aparentemente a un motivo amoroso, a su vez deviene objeto de engaño -y/o de sometimiento- para las mujeres que la reciben. En tal sentido, consideraremos los lexemas y sintagmas mediante los que el poeta alude a dicha fruta, y señalaremos sus eventuales vínculos intertextuales con otras obras de la tradición clásica, para relevar aquellos usos marcados que resulten significativos.

\section{Abstract}

Ovid uses the apple as a symbolic element to refer to the love stories of Cyddipe and Acontius, in Heroides 20 and 21, and those of Atalanta and Hippomenes, in

\section{Keywords}

Ovid; myth; apple; deceit; intertextuality Metamorphoses 10 (560-707). In both cases the union of the couples is caused by a trick or stratagem, whose trigger -the apple- conditions the representation of the female figures. In this paper we will address the symbolic resignification of the apple in both myths, not only as a plot component, but also as a structural element of the Ovidian narratives. There, it has are an ambiguous value, since although it apparently stands for a love motive, it also becomes an object of deception -and/or submission- for the women who receive it. In this sense, we will consider the lexemes and syntagms through which the poet alludes to this fruit, and we will point out its possible intertextual links with other works of the classical tradition, in order to reveal those uses that are significant to this end. 
Este trabajo, enmarcado en un proyecto más amplio, ${ }^{1}$ se orienta a discernir si las distintas calificaciones atribuidas a la manzana condicionan o no los destinos de las figuras femeninas en los mitos de Cídipe y Atalanta, por parte de las voces enunciativas construidas en Heroidas y en Metamorfosis de Ovidio.

Para abordar el tema partimos del simbolismo de la manzana ${ }^{2}$ tanto en la epístola 20, en la que Aconcio narra la conquista de Cídipe, como en Metamorfosis 10, cuando Hipómenes seduce a Atalanta. En ambos casos, esta fruta, recurrentemente aludida en la literatura griega y latina, remite al tradicional tópico del amor. Sin embargo, consideramos que su particular uso, en tanto estrategia que posibilita la conquista de una joven, habilita otras lecturas de los mitos en los que aparece incluido. Es decir, mientras que por un lado guarda relación con el amor, por otro, reviste también un carácter negativo, como se deduce a partir de la perspectiva enunciativa femenina de la Heroida $21 .^{3}$

Para el estudio de la resignificación de la manzana en los mitos mencionados, realizamos una lectura intertextual a partir de los planteos teóricos de Fowler (1997), para quien "[the] intertextual reading, far from being a formalist method isolating texts within literature, is essentially ideological. To say that this text is relevant but not this text is not to discover literary system but to construct it" (p. 25). Proponemos, en consecuencia, una interpretación del simbolismo de la fruta y su vinculación con la representación de las figuras femeninas a partir de la identificación de algunas de las referencias intra e intertextuales, que constituyen un componente inherente de la poética ovidiana. ${ }^{4}$ Los siguientes lexemas y sintagmas referidos a la manzana en los mitos de Cídipe y de Atalanta conforman los campos semánticos seleccionados para el análisis de este trabajo: volubile malum (epist. 20.209), aurea mali felicis imago (epist. 20.237), aurea poma (met. 10.650), nitidi pomi (met. 10.666) y aurum volubile (met. 10.667).

Si bien malum ${ }^{5}$ se utiliza como término genérico para designar la mayor parte de las frutas (excepto las nueces, Littlewood, 1968, p. 147 y ss.), en estas dos narraciones el elemento de vinculación es, puntualmente, una manzana. ${ }^{6}$ Asimismo, la palabra más usada para referirse a este fruto, principalmente en la carta 21, es pomum y no malum; en este caso, el primero debe entenderse como un hiperónimo del segundo.

1. Este trabajo se enmarca en el PI 17H014, titulado "Dimensiones monstruosas de la épica y de sus héroes en Heroidas de Ovidio" y financiado por la SGCyT de la Universidad Nacional del Nordeste, en el que participo como becaria investigadora.

2. Para profundizar el estudio de ese simbolismo, cf. los trabajos de Foster (1899), sobre la manzana como muestra de amor; de Gaidoz (1902), sobre la preferencia por esta fruta en contextos amorosos; y de McCartney (1925), sobre la manzana como símbolo de fertilidad en diferentes culturas de la Antigüedad. Los estudios de Littlewood (1968) y Matthey $(2013,2015)$ presentan un catálogo exhaustivo del simbolismo de la manzana en la literatura clásica, especialmente en relación con las manzanas doradas del jardín de las Hespérides.

3. Coincidimos con Camino Plaza (2016), cuando afirma que Ovidio construye una marca indeleble en la elegía amorosa porque les da voz a mujeres de la mitología o la historia que antes no la habían tenido, permitiendo así confrontar dos miradas genéricamente distintas a partir de una misma historia o mito.

4. Con respecto a la importancia de la intertextualidad en el estudio del corpus ovidiano, cf., entre otros, Casali (2009), Kennedy (1984), Miller (1993) y Barchiesi (1993).

5. Cf. OLD, s.v. malum ${ }^{2}$ : "A term converting originally most soft-skinned tree fruit, but later normally specifying an apple"; OLD s.v. pomum: "tree fruit", "a fruit"; ThLL.10.2601.40-73; y OLD, s.v. malum "an apple, i. e. any tree-fruit fleshy on the outside, and having a kernel within (opp. nux); hence, applied also to quinces, pomegranates, peaches, oranges, lemons, etc.".

6. Cf. McCartney (1925, p. 73). 
En la epístola 20 las palabras de Cídipe establecen la relación con Atalanta a partir de esa fruta: "A Cídipe la engañó una fruta, a la hija de Esqueneo la engañó una fruta (Cydippen pomum, pomum Schoeneida cepit; epist. 21.123): tú ahora serás sin duda un segundo Hipómenes (tu nunc Hippomenes scilicet alter eris!; epist. 21.124)". A su vez, en Metamorfosis Ovidio utiliza el término pomum (nitidi pomi; met. 10.666) para señalar el objeto con el que fue engañada Atalanta. Observamos, en consecuencia, que la relación entre Cídipe y Aconcio y Atalanta e Hipómenes se entrelaza a partir de la presencia de esa fruta, relacionada con Afrodita, la conquista amorosa, la fertilidad, el matrimonio y el erotismo. ${ }^{7}$

Según relata Calímaco en el poema 66 de los Aitia (frs. 67-75) -probable fuente de Ovidio-, ${ }^{8}$ Aconcio conoce a Cídipe cuando visita Delos con su madre y, cautivado por su belleza, se enamora de ella y le lanza una manzana con un juramento como inscripción. Cídipe la lee de pie, en el templo de Diana, e involuntariamente jura casarse con Aconcio. Al regresar a su casa, tres veces se enferma en la víspera de su matrimonio con otro prometido; ante la imposibilidad de concretar las nupcias, su padre consulta el oráculo de Apolo, y este le advierte que un juramento impide a su hija casarse con alguien que no sea el joven Aconcio. Es justamente en este momento de la secuencia narrativa ${ }^{9}$ en el que Ovidio ubica el intercambio epistolar entre ambos personajes: en el cuarto día posterior a las truncadas bodas de Cídipe (Kenney, 1970, p. 391).

Como en las otras cartas dobles, ${ }^{10}$ es el joven quien inicia el intercambio epistolar; en este caso, para reclamarle a Cídipe el cumplimiento del juramento realizado en el templo de Diana, y para describir la estratagema de la manzana, a la que había recurrido para engañarla. En los versos finales el enunciador narra el momento en que contempla por primera vez a la doncella, por cuya belleza queda clavado (fixis in tua membra genis, 20.206), y le arroja, cautivado, una manzana con palabras traicioneras.

El adjetivo usado para describir la fruta, volubile, indica que la manzana llega girando y rodando como un trompo, pero también que se trata de una acción que muda el orden y altera el curso de las cosas. ${ }^{11}$ Si bien el obsequio está relacionado con la conquista y la seducción, acaso porque esta fruta resulta una de las más nobles por su color, olor y textura (Littlewood, 1968, p. 181), volubile sugiere también un cambio de fortuna ya que el obsequio pasa de ser un motivo amoroso a un engaño, produciendo una alteración en el destino de ambos jóvenes. En tal sentido, existe la posibilidad de interpretar el pasaje a partir de la homonimia entre manzana (malum) y desgracia (malum), ya que el cambio de suerte padecido por Cídipe permite el juego de significados propuestos:

7. Cf. Foster (1899) y Littlewood (1968).

8. La historia aparece también en Calímaco (Pfeiffer, 67-75), Catulo (65; 19-24), Propercio (1.23.4, 1.18), Ovidio (rem. 381-382; ars 1. 457; trist. 3.10.73) y en Aristeneto (1.10).

9. Sobre el momento compositivo de las cartas en el marco de la narración mítica, cf. Casali (2009); Camino Plaza (2016)

10. Con respecto a la autenticidad y la datación de las cartas dobles, cf. los estudios de Clark (1908), d'Elia (1957); Tracy (1971); Barchiesi (1999); Beck (1997); Rosati (1996) y la introducción de Kenney (1996); mientras estos autores consideran auténticos los tres pares de cartas, Courtney (1965 y 1998) niega que la autoría de las mismas pertenezca a Ovidio.

11. Verg. Aen. 7.381-382: stupet inscia supra / impubesque manus mirata volubile buxum; y la descripción de los movimientos de la reina Amata como el otro polo de la comparación. 
Postmodo nescioqua venisse volubile malum

(Ov. epist. 20.209) $)^{12}$

Luego, no sé de dónde había llegado la manzana en movimiento

Luego, no sé por dónde había llegado la desgracia en movimiento

En circunstancias similares, según la narración incluida por Ovidio en el libro 10 de Metamorfosis, Hipómenes engaña a Atalanta. Allí, la historia, que es narrada por Venus a Adonis, forma parte de un complejo entramado de voces enmarcadas. El relato de la diosa forma parte de otro mayor, cantado por Orfeo, personaje que evoca, como arquetipo poético, la tarea compositiva de Ovidio. ${ }^{13}$

Las estratagemas usadas por Aconcio e Hipómenes presentan las mismas características en las dos historias: las manzanas resultan provistas por una deidad, son utilizadas para conquistar muchachas a través de un engaño, y también son adjetivadas como volubila:

Obstipuit virgo nitidique cupidine pomi

declinat cursus aurumque volubile tollit

(Ov. met. 10.666-667) $)^{14}$

Quedó atónita la doncella y, codiciando la resplandeciente fruta, desvía su camino y recoge el oro en movimiento.

En ambos casos, el lexema indica un doble movimiento: por un lado, remite a un desplazamiento físico, ya que la manzana llega hasta las jóvenes rodando y, por otro, conlleva un efecto que se concreta cuando su movimiento se detiene por la intervención de las muchachas.

En el citado pasaje de Metamorfosis, el verbo declino revela el impacto que causa la aparición de las manzanas en la carrera de Atalanta, pues la joven detiene el ritmo de su marcha, obnubilada por la belleza del objeto que se presenta ante sus ojos (obstipuit virgo). Además, el uso del verbo obstipesco, ubicado al comienzo del dístico, contribuye a reforzar la idea de que la joven, paralizada, no puede seguir avanzando por la interferencia de un deseo superior, motivado por la intervención de Venus, diosa que representaba un potencial peligro para una joven que, como Atalanta, era una virgen consagrada a Diana. En este sentido, Atalanta es, al igual que Cídipe, copartícipe involuntaria de la cacería que la tiene como presa: mientras la primera se demora en la carrera y recoge la manzana, la otra lee el juramento inscripto en la superficie de la fruta.

No obstante, la visión de Aconcio acerca de la estratagema difiere de la percepción de la propia Cídipe, ya que él cierra su carta presentando nuevamente la imagen de la manzana como el nexo que vincula a los amantes. Además, se refiere a la fruta en términos votivos, señalando lo que representa para él la dorada imagen (aurea imago) ${ }^{15}$ de la manzana feliz (mali felicis). El adjetivo felix concentra el sentido final de la epístola y, en consecuencia, realza el valor positivo que le otorga el muchacho:

12. Seguimos la edición de Kenney (1996). Todas las traducciones nos pertenecen.

13. Cf. Casali (2009, p. 350).

14. Seguimos la edición de Anderson (1998). Todas las traducciones nos pertenecen.

15. La presencia del adjetivo aurea remite también a "aurum volubile”, fortaleciendo así la relación entre ambas historias. 
quod si contigerit, cum iam data signa sonabunt,

tinctaque votivo sanguine Delos erit,

aurea ponetur mali felicis imago,

causaque versiculis scripta duobus erit:

"effigie pomi testatur Acontius huius

quae fuerint in eo scripta, fuisse rata”.

(Ov. epist. 20.235-240)

Y si esto sucediera, cuando suenen las señales dadas y Delos esté teñida de sangre votiva se ofrecerá la imagen dorada de la manzana feliz, y se escribirá el motivo en dos versitos: "Con la efigie de esta manzana Aconcio da fe del cumplimiento de lo que en ella estaba escrito".

Notamos, a su vez, la implícita alusión a la descripción del limón en VERG. georg. 2.126-12716 (tardumque saporem/felicis mali) que refuerza las diferencias entre las perspectivas de Cídipe y Aconcio. En este pasaje malum se refiere al cítrico cuyo sabor ácido se relaciona con las manzanas doradas extraídas del jardín de las Hespérides, usadas por Hipómenes para conquistar a Atalanta. A su vez, felix, ${ }^{17}$ adjetivo empleado con el sentido de "fértil", "fructífero", señala que es un fruto de ambivalentes características, ya que si bien su sabor es poco agradable, se lo usa para curar algunas enfermedades o para contrarrestar ciertos venenos. De acuerdo con esta acepción, podríamos interpretar también que la manzana, además de feliz, resulta fértil, es decir, fructífera y favorable para Aconcio, pues con ella logra conquistar a Cídipe.

En la Heroida 20 el sintagma felicis mali usado por Aconcio habilita también otras lecturas, como por ejemplo la ya propuesta aquí con relación a postmodo nescioqua venisse volubile malum (Ov. epist. 20. 209). El pasaje se puede interpretar a partir de la ambigüedad del término malum, como la expresión de dos significados probables: "manzana feliz", pero también "desgracia feliz", con su carga de oxímoron. ${ }^{18}$

Para Cídipe, en cambio, la manzana representa un doble efecto negativo: por un lado, el regalo afecta notablemente su salud, provocando la presencia de signos visibles de deterioro en su cuerpo, y por otro, la obliga a contraer nupcias con Aconcio. Al describir los cambios en su estado físico desde el momento en el que recibe la fruta, ella contrasta el color de la manzana con su propia palidez como señal de enfermedad: ${ }^{19}$

concidimus macie, color est sine sanguine, qualem

in pomo refero mente fuisse tuo,

candida nec mixto sublucent ora rubore.

(Ov. epist. 21.215-217)

Me muero de la delgadez, he perdido el color de la sangre, como el que tenía, lo recuerdo en mi mente, tu manzana, y no luce cándido mi rostro matizado de rojo.

16. Seguimos la edición de Mynors (1972).

17. Cf. $O L D$, s.v. felix : "Fruit-bearing, fruitful, fertile, productive".

18. Cf. Kay (2006, p. 195).

19. La palidez, vinculada con el amor, pero también con la enfermedad y la muerte, es recurrente en la literatura latina (cf. Moreno Soldevilla, 2011, p. 402). 
La descripción de los colores que luce su semblante, ${ }^{20}$ en la epístola 21 , le sirve a la joven para realzar su propia percepción de la historia. La blancura (candida $)^{21}$ de su rostro reafirma su belleza como virgen, vinculada íntimamente con la pureza y la inocencia; Aconcio, en cambio, lo había descripto antes en términos erotizados: quique subest niveo ${ }^{22}$ lenis in ore rubor (20.120). El adjetivo niveus, en relación con rubor, connota sexualmente el pasaje. ${ }^{23}$ Cabe aclarar que se trata también del color de las víctimas sacrificadas a los dioses Olímpicos. Consideramos, en este sentido, que el uso del calificativo habilita también un paralelismo entre Cídipe y las vírgenes ofrecidas a los dioses, ya que la joven debe "sacrificar" sus propios deseos a los intereses de Aconcio.

El contraste entre ambas percepciones resulta evidente: mientras Cídipe se autoproclama una víctima inocente, las palabras de Aconcio se concentran en su propio deseo por el rostro de la amada.

Frente a otros análisis, en los que se considera que la historia de Cídipe y Aconcio tiene un final feliz (cf. Kenney, 1996; Ramírez de Verger Jaén, 2010), creemos que el texto permite otra lectura, ya que Ovidio recurre a un sintagma que utiliza también para referirse al absoluto estado de dolor de Niobe, en el libro 6 de Metamorfosis, cuando Apolo y Diana matan a todos los hijos que esta había tenido con Anfión, para castigar su soberbia y su actitud desafiante hacia Latona. Mediante el uso de la misma expresión, se construye un paralelismo entre el aspecto de Cídipe y el de Níobe, próxima a la muerte: ${ }^{24}$

(...) nullos movet aura capillos,

in vultu color est sine sanguine, lumina maestis

stant inmota genis, nihil est in imagine vivum.

(Ov. met. 6.303-305)

La brisa no mueve ninguno de sus cabellos, su rostro ha perdido el color de la sangre, sus ojos están inmóviles en sus tristes mejillas, nada está vivo en su figura.

La falta de color en el rostro de Cídipe, opuesto al vivo rojo de la manzana, remarca el efecto negativo de la fruta sobre la muchacha. El contraste entre luminosidad y palidez habilita, a su vez, la identificación de dos perspectivas: una masculina y otra femenina. Si para Aconcio la manzana representa un destino feliz, para Cídipe la fruta y la muerte se encuentran equiparadas; la segunda es una consecuencia metafórica de la primera, como se observa en el siguiente pasaje, donde la doncella relaciona su destino con el de Atalanta, precisamente a partir de la fruta:

\footnotetext{
Cydyppen pomum, pomum Schoeneida cepit

tu nunc Hippomenes scilicet alter eris!
}

(Ov. epist. 21.123-124)

20. Respecto del uso de los colores en la elegía, cf. Barolsky (2003) y Dyson (1999); sobre el campo semántico del color en la lengua latina, cf. André (1949).

21. Cf. $O L D$ s. v. candidus ${ }^{5}$ : "Fair skinned, fair (usu.implying beauty), $O L D$ s.v candidus $s^{8 b}$ : Morally pure, innocent"; y $T h L L$ 3.244.43 "benevolus, bonus, simplex, sincerus".

22. Cf. OLD s.v. niveus": "Snow-white, snowy", y OLD s.v niveus ${ }^{2 \mathrm{~b}}$ : "As the colour of victims sacrificed to the Olympian deities".

23. Cf. Barolsky (2003, p. 53-54).

24. Cf. también Ov. am.1.7.51-42: astitit illa amens albo et sine sanguine vultu, / caeduntur Pariis qualia saxa iugis. 
A Cídipe engañó una fruta, a la hija de Esqueneo la capturó una fruta: tú ahora serás, sin duda, un segundo Hipómenes.

Ambas jóvenes son engañadas por la intervención de un elemento que favorece la manipulación y altera la condición de quienes lo reciben. Por otra parte, el peso semántico del dístico recae en el verbo capio, cuyo uso es recurrente en la tradición épica y revela el carácter aciago del destino que les espera a las mujeres, generalmente sometidas a los héroes por medio de la fuerza, o bien consideradas como trofeos de guerra. En este sentido, Cídipe se reconoce explícitamente como presa de un artificio (praeda; 21.122) al comparar su situación con la de Atalanta.

Los últimos versos de la epístola 21 presentan indiscutibles lagunas (Kenney, 1996, p. 246), sin embargo el sentido general del fragmento refuerza la idea de que Cídipe ha aceptado finalmente el matrimonio, obligada a cumplir el juramento inscripto en la manzana, sin que esto represente una expresión de su propio deseo. La presencia del participio libens ${ }^{25}$ señala la observancia religiosa que subyace tras su resignada aceptación de la boda con Aconcio, entendida solo como imperativa contraparte del juramento realizado:

doque libens victas in tua vota manus;

fassaque sum matri deceptae foedera linguae

lumina fixa tenens plena pudoris humo

(Ov. epist. 21.240-242)

Y entrego con voluntad las manos vencidas a tus deseos; y he confesado a mi madre, con los ojos llenos de vergüenza fijos en el suelo, el juramento que hizo mi lengua engañada.

A su vez, el uso del participio victas refuerza la idea de que se trata de una acción acatada más por obligación que por convicción o decisión. La vergüenza en sus ojos (lumina plena pudoris) es signo, como señala Kenney (1996, p. 246), de que la joven ha comprendido que fue seducida mediante un cortejo violento y sin escrúpulos.

\section{Conclusiones}

A través del uso de los sintagmas malum volubile (epist. 20.209) / aurum volubile (met. 10.667), aurea felicis mali imago (epist. 20.237), y aurea poma (met. 10.650) Ovidio activa la correlación simbólica entre las historias de Cídipe y Atalanta, mientras el uso de la expresión color est sine sanguine (epist. 21.215 y met. 6.304) le permite vincular a Cídipe con otra figura mitológica próxima a la muerte, Níobe. La presencia de estos elementos nos condujo a reinterpretar el simbolismo de la manzana como estrategia de seducción en ambas narraciones míticas, mostrando que guarda relación no solo

25. Cf. Verg. Aen. 3.438: cane vota libens; cuando Heleno ordena a Eneas hacer votos a Juno para aplacar su cólera y poder llegar finalmente al Lacio. Kenney (1996) plantea que Cídipe finalmente cede al matrimonio más por observancia religiosa, indicada por el participio libens, que por placer. A partir de la relación del pasaje con el texto virgiliano, todo parece indicar que Cídipe intenta evitar la ira de Diana, en cuyo templo había jurado casarse con Aconcio; por otra parte, es el propio Aconcio quien le aconseja no desafiar a la diosa (Parce movere feros animosae virginis arcus / mitis adbuc fieri, si patiare, potest; ( parce, precor, teneros corrumpere febribus artus, 20.117-119). 
con la dimensión amorosa de la conquista, sino también con el adverso destino que el contacto con la fruta representa para las jóvenes.

Además, hemos podido distinguir aquellos lexemas y sintagmas que le otorgan a la manzana, fruto que configura el destino de Cídipe y Atalanta, un valor negativo en las dos narraciones ovidianas. Su caracterización por parte de Cídipe, en la Heroida 21, contrasta con la que Aconcio le atribuye en la Heroida 20. Mientras que la manzana representa un objeto de seducción y conquista para la figura masculina, condiciona la libertad de la joven, quien no le atribuye un valor positivo. Claramente concebida en la tradición como un obsequio para halagar a las doncellas, la manzana deviene en las epístolas un objeto que oculta el sometimiento y el cambio de fortuna para Cídipe y Atalanta.

Finalmente, sostenemos que las alusiones intertextuales modifican la tradicional representación de la fruta como motivo amoroso y, a partir de la voz femenina, dotan de nuevos significados al obsequio. La particular visión de Cídipe sobre la manzana habilita una mirada distinta sobre la estratagema usada por Aconcio para conquistarla y, a la vez, nos invita a resignificar la historia de Atalanta desde esa perspectiva.

\section{Referencias bibliográficas}

Anderson, W. (ed.). (1998). P. Ovidii Nasonis Metamorphoses. Leipzig, Teubner.

André, J. (1949). Étude sur les termes de couleurs dans la langue latine. Paris, Klincksieck.

Barchiesi, A. (1993). Future Reflexive: Two Modes of Allusion and Ovid's Heroides. Harvard Studies in Classical Philology, 95, 33-365. https://bit.ly/34Du7Gz

Barchiesi, A. (1999). Vers une histoire à rebours de l'élégie latine: les Héroïdes 'doubles' (16-21). Electronic Antiquity, 5 (1), 1-16. https://bit.ly/3lpPCAI

Barolsky, P. (2003). Ovid's Colors. Arion, 3 (10), 51-56. https://bit.ly/2EAVlm9

Beck, M. (1997). Die Epistulae Heroidum XVIII und XIX des Corpus Ovidianum. Paderborn, Echtheitskritische Untersuchungen.

Camino Plaza, L. (2016). Las voces femeninas en las Heroidas de Ovidio. Nuevas aproximaciones a la elegía de mujeres. ArtyHum, 20, 132-143. https://bit.ly/31ypWtr

Casali, S. (2009). Ovidian Intertextuality. En: Knox, P. (edit.), Annali della Facoltà di Lettere e Filosofia di Napoli (pp. 341-354). Napoli, Blackwell.

Clark, S. (1908). The Authorship and the Date of the Double Letters in Ovid's Heroides. Harvard Studies in Classical Philology, 19, 121-155. https://bit.ly/31xfqL1

Courtney, E. (1965). Ovidian and non-Ovidian Heroides. Bulletin of the Institute of Classical Studies, 1, 63-66. https://bit.ly/2YEqzQD

Courtney, E. (1998). Echtheitskritik: Ovidian and non-Ovidian Heroides again. The Classical Journal, 93 (22), 157-166. https://bit.ly/2G7IYip

D’Elia, S. (1957). Problemi Ovidiani. Cronologia e autenticità di Her. XVI-XXI. Annali della Facoltà di Lettere e Filosofia di Napoli, 7, 57-91. 
Dyson, J. (1999). Lilies and violence: Lavinia's blush in the song of Orpheus. Classical Philology, 94 (3), 281-288. https://bit.ly/2QtCLPp

Foster, B. (1899). Notes on the Symbolism of the Apple in Classical Antiquity. Harvard Studies in Classical Philology, 10, 39-55. https://bit.ly/31mZfQt

Fowler, D. (1997). On the Shoulders of Giants: Intertextuality and Classical Studies. Materiali e discussioni per l'analisi dei testi classici, 39, 13-34. https://bit.ly/3b3u7Rt

Gaidoz, H. (1902). La réquisition d'amour et le symbolisme de la pomme. Annuaire de l'École Pratique des Hautes Études, IVème section, Sciences historiques et philologiques, 5-33. https://bit.ly/2EnWLRn

Kay, N. (2006). Epigrams from the Anthologia Latina. Text, translation and commentary. London, Duckworth.

Kennedy, D. (1984). The Epistolary Mode and the First of Ovid's Heroides. The Classical Quarterly, New Series, 34 (2), 413-422. https://bit.ly/34B6Hlb

Kenney, E. (1970). Love and legalism. Ovid 20 and 21. Arion, 9, 388-414. https://bit.ly/34AY2z7

Kenney, E. (1996). Ovid: Heroides XVI-XXI. New York, Cambridge University Press.

Littlewood, A. (1968). The Symbolism of the Apple in Greek and Roman Literature. Harvard Studies in Classical Philology, 72, 147-181. https://bit.ly/3gzC8yG

Matthey, P. (2013). Les pommes d'amour des Hespérides. Le jardin aux portes du soir. En: Barbu, D.; Borgeaud, P. y Volokhine, Y. (eds.), Mondes clos. Cultures et jardins (pp. 139-163). Lausana, Infolio. https://bit.ly/3lqgCQs

Matthey, P. (2015). Les pommes d'amour des Hespérides (2ème partie). Dans les paradis païens. En: Borgeaud, P., Meylan, N. y Volokhine, Y. (eds.), Mondes clos. Les îles. Goillon (pp. 158-194). Lausana, Infolio. https://bit.ly/3jISqHx

McCartney, E. (1925). How the Apple Became the Token of Love. Transactions and Proceedings of the American Philological Association, 56, 70-81. https://bit.ly/31zl07T

Miller, J. (1993). Ovidian Allusion and the Vocabulary of Memory. Materiali e discussioni per l'analisi dei testi classici, 30, 153-164.

Mynors, R. (1969). Vergilii Maronis Opera. Oxford, Oxford University Press.

Moreno Soldevila, R. (ed.). (2011). Diccionario de motivos amatorios en la literatura latina (siglos III a.C.-II d.C). España, Universidad de Huelva.

Mozley,J.y Goold, G. (1979). Ovid in Six Volumes. Vol. 2: Ars Amatoria. Cambridge, Harvard University Press.

OLD = The Oxford Latin Dictionary. Oxford, Oxford University Press.

Ramirez de Verger Jaén, A. (2010). Ovidio. Heroidas. Madrid, Akal.

Rosati, G. (1996). Ovidii Nasonis Heroidum Epistulae XVIII-XIX. Leander Heroni Hero Leandro. Firenze, Le Monnier.

ThLL = Thesaurus Linguae Latinae, Leipzig, Stuttgart.

Tracy, V. (1971). The Authenticity of Heroides 16-21. The Classical Journal, 66 (4), 328-330. https://bit. ly/2Qv0hvz 
*Abigail Luz Marina Méndez es Licenciada en Letras (Universidad Nacional del Nordeste). Actualmente, es Profesora JTP en el Seminario de Literatura y Otros Discursos Sociales I y Auxiliar docente en el Seminario Taller de Literatura de la Facultad de Humanidades (UNNE). Es integrante del PI 17H014: "Dimensiones monstruosas de la épica y de sus héroes en Heroidas de Ovidio" (Secretaría General de Ciencia y Técnica, UNNE), becaria del CIN (2019-2020) y adscripta a Lengua y Cultura Latinas (2018-2020).

RECEPCIÓN: $16 / 06 / 2020$

ACEPTACIÓN: 17/08/2020 